\title{
Extremal binary matrices without constant 2-squares
}

\author{
Roland Bacher AND Shalom Eliahou
}

\begin{abstract}
In this paper we solve, by computational means, an open problem of Erickson: denoting $[n]=\{1, \ldots, n\}$, what is the smallest integer $n_{0}$ such that, for every $n \geq n_{0}$ and every 2-coloring of the grid $[n] \times[n]$, there is a constant 2 -square, i.e. a $2 \times 2$ subgrid $S=$ $\{i, i+t\} \times\{j, j+t\}$ whose four points are colored the same? It has been shown recently that $13 \leq n_{0} \leq \min \left(W(2,8), 5 \cdot 2^{2^{40}}\right)$, where $W(2,8)$ is the still unknown 8th classical van der Waerden number. We obtain here the exact value $n_{0}=15$. In the process we display 2-colorings of $[13] \times \mathbb{Z}$ and $[14] \times[14]$ without constant 2 -squares, and show that this is best possible.
\end{abstract}

AMS 2010 SUBJECT CLASSIFICATION: 05D10, 11B75.

\section{Introduction}

For a positive integer $n$, denote $[n]=\{1, \ldots, n\}$. In his lovely book, Martin J. Erickson posed the following problem [9, p. 36].

Open Problem 4. Find the minimum $n$ such that if the $n^{2}$ lattice points of $[n] \times[n]$ are two-colored, there exist four points of one color lying on the vertices of a square with sides parallel to the coordinate axes.

The author further hints in Exercise 4.24 of $[9$, p. 70$]$ that the least such $n$ satisfies $n \leq 9\left(2^{81}+1\right)\left(2^{\left(2^{81}+1\right)^{2}}+1\right)$.

Let us slightly rephrase the problem. A 2-square in the grid $[n] \times[n]$ is a $2 \times 2$ subgrid of the form

$$
S=\{i, i+t\} \times\{j, j+t\}
$$

for some index $t \geq 1$. It follows from a theorem of Gallai (see Section 5.2) that if $n$ is large enough, then for any 2-coloring

$$
c:[n] \times[n] \rightarrow\{0,1\},
$$


the grid $[n] \times[n]$ must contain a monochromatic 2 -square, i.e. whose four points are all colored 0 or 1 under $c$. The smallest integer $n$ for which this occurs is denoted $R_{2}(S)$ in [2]. In that paper, it is shown that

$$
13 \leq R_{2}(S) \leq \min \left(W(2,8), 5 \cdot 2^{2^{40}}\right),
$$

where $W(2, l)$ is the classical $l$ th van der Waerden number. Recall that $W(k, l)$ is the smallest $m \geq 1$ for which any $k$-coloring of $[m]$ contains a monochromatic subsequence forming an arithmetic progression of length $l$.

Perhaps surprisingly in view of such bounds, in this paper we solve Erickson's problem by computer and obtain the exact value

$$
R_{2}(S)=15
$$

The situation may be compared to that of van der Waerden numbers. The best known general upper bound for $W(2, l)$, due to Gowers [10], is

$$
W(2, l) \leq 2^{2^{2^{2^{l+9}}}}
$$

However, the actual values of $W(2, l)$ for $3 \leq l \leq 6$, namely 9, 35, 178 and 1132 , respectively, are much smaller than that. The value $W(2,6)=1132$ has been obtained recently by clever reductions and massive parallel computations with a specially designed SAT solver [13]. At the time of writing, the numbers $W(2, l)$ are unknown for $l \geq 7$. See [11] for a wealth of papers on Ramsey theory.

\subsection{Erickson matrices}

For convenience, we shall rather adopt the language of binary matrices, i.e. matrices with coefficients in the 2-element field $\mathbb{F}_{2}$. As for grids, a 2-square in a matrix $A$ is a $2 \times 2$ submatrix $S$ with row indices $\{i, i+t\}$ and column indices $\{j, j+t\}$ for some $t \geq 1$. In this case, we say that $S$ is of span $t+1$.

Definition 1.1. An Erickson matrix is a binary matrix containing no constant 2-squares.

We shall actually refine our claimed solution $R_{2}(S)=15$ as follows. (See also Theorem 4.1 below.)

Theorem 1.2. There exist Erickson matrices of sizes ${ }^{1} 13 \times \infty$ and $14 \times 14$. There exist no Erickson matrices of size $14 \times 15$.

\footnotetext{
${ }^{1}$ Throughout the paper, the symbol $\infty$ shall stand for $\aleph_{0}$, the smallest infinite cardinal.
} 
In other words, every 2 -coloring of the grid [14] $\times[15]$ contains a monochromatic 2-square. Conversely, there do exist 2 -colorings of the grids $[13] \times \mathbb{Z}$ and $[14] \times[14]$ without monochromatic 2-squares.

The paper is organized as follows. In Section 2, we investigate Erickson matrices with special properties, such as antisymmetry, symmetry, and with constant first row, and obtain the maximum possible size in each case. In Section 3, we explain the backtracking algorithm we have used in the general case. In Section 4, we exhibit an extremal Erickson matrix of size $13 \times \infty$, and give a parametric description of all square Erickson matrices of size 14 up to automorphisms. This allows us to show that there are no Erickson matrices of size $14 \times 15$. In the last section, we discuss related structures such as Erickson tori, Erickson triangles and higher dimensional analogues. We conclude with some historical remarks about a theorem of Gallai preventing such objects of being too large.

Throughout the paper, we shall interchangeably display binary matrices as plain matrices or as black-and-white boards, with white for 0 and black for 1 . For $\epsilon \in \mathbb{F}_{2}$, we shall denote $\bar{\epsilon}=1-\epsilon$ and call this operation the flip. Thus $\overline{0}=1$ and $\overline{1}=0$.

\section{Three special cases}

This work started with the objective of constructing a large Erickson matrix by computer. In order to cut down the volume of computations, we chose to focus on skew matrices with zero diagonal (see below). The first surprise occurred: even though millions of such matrices were found in size $n \times n$ with $n \leq 10$, only very few remained for $n=11$ already, and none for $n \geq 12$. This gave a strong hint that in the general case, the maximum size of an Erickson matrix would not be too far from 11, and be actually reachable by exhaustive computer search. This turned out to be true: the maximum size of a square Erickson matrix is found here to be $n=14$.

In this section, we study extremal square Erickson matrices in three special cases: skew, symmetric, and with constant first row. The maximum admissible size in each case turns out to be 11, 8 and 9, respectively (see below).

The construction method by computer in these instances does not need to be very sophisticated. In its most basic version, for each $n \geq 1$, one stores the set of Erickson matrices of size $n$ with the given property, up to some automorphisms to reduce the load. A somewhat simple extension process then allows to pass from $n$ to $n+1$. One useful trick consists in completing constant elbows. This means that, whenever an unassigned entry $a_{i j}$ lies in 
a 2-square whose three other entries are equal to $\epsilon \in \mathbb{F}_{2}$, then $a_{i j}$ must be set to $\bar{\epsilon}=1-\epsilon$.

The reader may sense the importance of this trick by trying it out on this partially filled matrix:

$$
A=\left(\begin{array}{ccccc}
0 & 0 & 1 & 1 & 1 \\
1 & 0 & 1 & 0 & \square \\
0 & 0 & 0 & 0 & \square \\
0 & 1 & 1 & 0 & \square \\
0 & \square & \square & \square & 0
\end{array}\right) .
$$

By completing constant elbows, a few seconds suffice to check by hand that there is a unique $5 \times 5$ Erickson matrix coinciding with the partial matrix $A$.

\subsection{Skew Erickson matrices}

We start with skew binary matrices, i.e. square matrices $A=\left(a_{i j}\right)$ over $\mathbb{F}_{2}$ satisfying $a_{j i}=\overline{a_{i j}}=1-a_{i j}$ for all $i \neq j$. No hypothesis is made on the diagonal entries $a_{i i}$. Those entries are in fact irrelevant in the present case for the Erickson property, as shown now.

Proposition 2.1. Let $A$ be a skew Erickson matrix. Denote by $B$ any matrix obtained from $A$ by flipping some diagonal entries. Then $B$ is also a skew Erickson matrix.

Proof. Set $A=\left(a_{i j}\right)$. We may assume that in $B$, a single diagonal entry of $A$ is flipped, say $a_{i i}$ is replaced by $\overline{a_{i i}}$. Of course, $B$ is skew since $A$ is. Assume that $B$ contains a constant 2 -square $S$. Then one of the four positions of $S$ is $(i, i)$. This position cannot be the NW or SE corner of $S$, for otherwise $S$ would also be skew, a contradiction. Therefore $(i, i)$ must either be the NE or SW corner of $S$, and hence $S$ is entirely contained in either the upper or the lower triangular part of $B$. Denote $S^{\prime}$ the symmetric image of $S$ under transposition. Then one of the entries of $S^{\prime}$ is still $\overline{a_{i i}}$ at position $(i, i)$ as in $S$, but the other three entries of $S^{\prime}$, being off-diagonal, are flipped with respect to the corresponding entries in $S$. This implies that $S^{\prime}$ is in fact a constant 2 -square in the original matrix $A$, a contradiction.

Consequently, when investigating skew Erickson matrices, we may freely assume that the diagonal is zero. This further reduces the volume of computations needed. Our computational result is that the largest possible size of a skew Erickson matrix is $n=11$. 
Theorem 2.2. There exist no skew Erickson matrices of size $n \geq 12$. In size $n=11$, there exist exactly 8 skew Erickson matrices with zero diagonal; up to automorphisms, they are all equivalent to the following one:

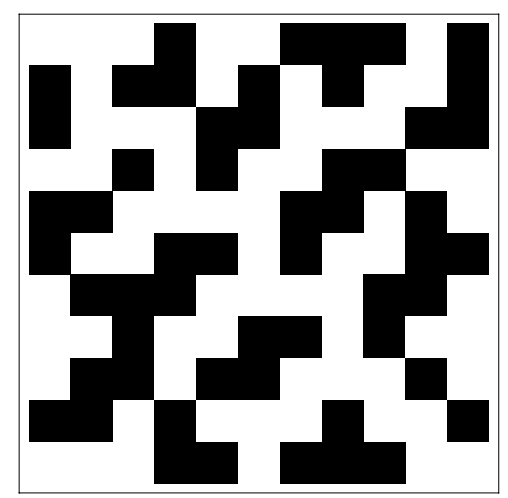

Note that the automorphism group of the set of skew Erickson matrices is of order 8. It is generated by transposition, half-turn rotation, and flipping of all the entries.

\subsection{Symmetric Erickson matrices}

The largest possible size for a symmetric Erickson matrix turns out to be 8. An example is given in Figure 1. The automorphism group of the set of symmetric Erickson matrices is the same as in the skew case.

Theorem 2.3. There are no symmetric Erickson matrices of size $n \geq 9$. There are exactly 152 symmetric Erickson matrices of size 8, partitioned into 38 orbits under the automorphism group.

More precisely, the 152 symmetric Erickson matrices of size 8 can be partitioned into five families $S_{1}, \ldots, S_{5}$, as follows.

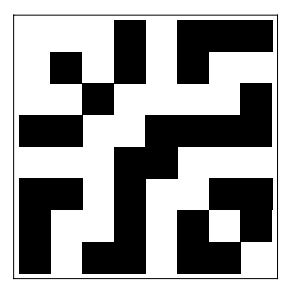

Figure 1: An extremal symmetric Erickson matrix of size 8. 
The family $S_{1}$ is given by

$$
S_{1}=\left(\begin{array}{cccccccc}
0 & 0 & x_{1} & 1 & 1 & 1 & 1 & x_{2} \\
0 & 1 & 0 & 0 & 1 & 0 & x_{3} & 0 \\
x_{1} & 0 & 1 & 0 & 0 & x_{4} & 1 & 0 \\
1 & 0 & 0 & 1 & x_{5} & 1 & 0 & 0 \\
1 & 1 & 0 & x_{5} & 0 & 1 & 1 & 0 \\
1 & 0 & x_{4} & 1 & 1 & 0 & 1 & 1 \\
1 & x_{3} & 1 & 0 & 1 & 1 & 0 & 1 \\
x_{2} & 0 & 0 & 0 & 0 & 1 & 1 & 1
\end{array}\right)
$$

subject to $\left(x_{1}, x_{2}, x_{4}\right) \neq(1,1,1)$. This family gives rise to 20 orbits. Eight orbits correspond to $x_{1}=0$ and come in pairs $\left(x_{1}=0, x_{2}, x_{3}, x_{4}, x_{5}\right),\left(x_{1}=\right.$ $\left.0,1-x_{2}, 1-x_{3}, 1-x_{4}, 1-x_{5}\right)$. The twelve solutions arising from $x_{1}=1$ give 12 more orbits which are all distinct.

The family

$$
S_{2}=\left(\begin{array}{cccccccc}
0 & 0 & 1 & 1 & 1 & 1 & 1 & x_{1} \\
0 & 1 & 0 & 0 & 1 & 0 & x_{2} & 0 \\
1 & 0 & 1 & 0 & 0 & x_{3} & 1 & 0 \\
1 & 0 & 0 & 1 & x_{4} & 1 & 0 & 0 \\
1 & 1 & 0 & x_{4} & 0 & 1 & 1 & 0 \\
1 & 0 & x_{3} & 1 & 1 & 0 & 1 & 0 \\
1 & x_{2} & 1 & 0 & 1 & 1 & 0 & 1 \\
x_{1} & 0 & 0 & 0 & 0 & 0 & 1 & 1
\end{array}\right)
$$

yields 8 orbits corresponding to the pairs $\left(x_{1}, x_{2}, x_{3}, x_{4}\right),\left(1-x_{1}, 1-x_{2}, 1-\right.$ $\left.x_{3}, 1-x_{4}\right)$.

The family $S_{3}$ is given by

$$
S_{3}=\left(\begin{array}{cccccccc}
x_{1} & 1 & x_{2} & 0 & 1 & 0 & 0 & x_{3} \\
1 & 0 & 1 & 0 & 1 & 0 & 1 & 1 \\
x_{2} & 1 & 0 & 1 & 1 & x_{4} & 1 & 0 \\
0 & 0 & 1 & 1 & 0 & 0 & 0 & 0 \\
1 & 1 & 1 & 0 & 0 & 1 & 1 & x_{5} \\
0 & 0 & x_{4} & 0 & 1 & 1 & 0 & 0 \\
0 & 1 & 1 & 0 & 1 & 0 & 1 & 0 \\
x_{3} & 1 & 0 & 0 & x_{5} & 0 & 0 & 1
\end{array}\right)
$$

subject to the inequalities

$$
\left(x_{1}, x_{2}\right) \neq(0,0), \quad\left(x_{1}, x_{3}\right) \neq(1,1), \quad\left(x_{3}, x_{4}\right) \neq(0,0), \quad\left(x_{3}, x_{5}\right) \neq(0,0) .
$$


This leaves 7 possibilities for the parameters giving rise to 7 orbits.

The next family

$$
S_{4}=\left(\begin{array}{cccccccc}
0 & 0 & 0 & 1 & 1 & 1 & 1 & 1 \\
0 & 1 & 0 & 0 & 1 & 0 & 0 & 1 \\
0 & 0 & 1 & 0 & x_{1} & 1 & 0 & 0 \\
1 & 0 & 0 & 1 & 0 & 1 & 0 & 1 \\
1 & 1 & x_{1} & 0 & 0 & 1 & 1 & 1 \\
1 & 0 & 1 & 1 & 1 & 0 & 0 & 1 \\
1 & 0 & 0 & 0 & 1 & 0 & 1 & 1 \\
1 & 1 & 0 & 1 & 1 & 1 & 1 & 0
\end{array}\right)
$$

gives rise to 2 orbits, and the last family represented by

$$
S_{5}=\left(\begin{array}{llllllll}
0 & 1 & 1 & 0 & 1 & 0 & 0 & 1 \\
1 & 0 & 1 & 0 & 1 & 0 & 1 & 1 \\
1 & 1 & 0 & 1 & 1 & 1 & 0 & 1 \\
0 & 0 & 1 & 1 & 0 & 0 & 0 & 0 \\
1 & 1 & 1 & 0 & 0 & 1 & 1 & 0 \\
0 & 0 & 1 & 0 & 1 & 1 & 0 & 0 \\
0 & 1 & 0 & 0 & 1 & 0 & 1 & 0 \\
1 & 1 & 1 & 0 & 0 & 0 & 0 & 1
\end{array}\right)
$$

gives rise to a unique orbit.

Summarizing, the orbits of these families sum up to $38=20+8+7+2+1$, representing all 152 symmetric Erickson matrices of size $8 \times 8$.

\subsection{Erickson matrices with constant first row}

We have found by computer that the largest size of a square Erickson matrix with constant first row is 9 . It is probably possible to obtain this result by a case-by-case analysis carried out by hand. Indeed, this is how the authors of [2] have shown that a square Erickson matrix with constant middle row has maximum size 7 , thereby proving $R_{2}(S) \leq W(2,8)$.

Theorem 2.4. For $n \geq 1$, let $\gamma(n)$ denote the number of square Erickson matrices of size $n$ with first row constant to 0 . Then $\gamma(n) \neq 0$ for $n \leq 9$ and 
$\gamma(n)=0$ for $n \geq 10$. The lexicographically smallest instance of size 9 is

$$
A=\left(\begin{array}{lllllllll}
0 & 0 & 0 & 0 & 0 & 0 & 0 & 0 & 0 \\
0 & 1 & 0 & 1 & 0 & 1 & 0 & 1 & 0 \\
0 & 0 & 1 & 1 & 0 & 0 & 1 & 1 & 0 \\
1 & 0 & 0 & 0 & 1 & 1 & 1 & 0 & 0 \\
0 & 1 & 0 & 1 & 1 & 0 & 1 & 0 & 1 \\
1 & 1 & 0 & 0 & 0 & 0 & 1 & 1 & 1 \\
1 & 0 & 1 & 0 & 1 & 1 & 0 & 1 & 0 \\
1 & 0 & 1 & 1 & 0 & 0 & 0 & 0 & 1 \\
0 & 1 & 1 & 0 & 0 & 1 & 1 & 0 & 1
\end{array}\right) .
$$

More precisely, we have

$$
(\gamma(1), \gamma(2), \ldots, \gamma(10))=(1,3,23,213,3754,35002,156188,79570,26276,0) .
$$

\section{The general case}

We start here our study of general square Erickson matrices. After a brief description of their automorphism group, we explain the algorithm we have used to construct them and to uncover their maximum possible size.

\subsection{Automorphisms}

The automorphism group $G$ of the set of square Erickson matrices is of order 16. It is isomorphic to the direct product

$$
G \cong D_{4} \times C_{2}
$$

where $D_{4}$ is the dihedral group of order 8 preserving the square, and $C_{2}$ is the group of order 2 flipping the entries, i.e. exchanging 0 and 1.

\subsection{The algorithm}

Consider binary variables $x_{1}, x_{2}, x_{3}, \ldots$ organized in an infinite array $X$ as follows:

$$
X=\left(\begin{array}{cccccc}
x_{1} & x_{3} & x_{6} & x_{11} & x_{18} & \ldots \\
x_{2} & x_{4} & x_{8} & x_{13} & \ldots & \ldots \\
x_{5} & x_{7} & x_{9} & x_{15} & \ldots & \ldots \\
x_{10} & x_{12} & x_{14} & x_{16} & \ldots & \ldots \\
x_{17} & x_{19} & \ldots & & \ddots & \\
\vdots & \vdots & & & & \ddots
\end{array}\right)
$$


Thus, for each $n \geq 1$, the first $n^{2}$ variables lie in an $n \times n$ square sub-array of $X$. We shall say that a variable is specialized when it is assigned a value in $\mathbb{F}_{2}$. Denote by $s(X)$ the set of all finite specializations of $X$, meaning that a finite number of the variables $x_{i}$ have been specialized. We now describe a simple forcing procedure

$$
\varphi: s(X) \rightarrow s(X)
$$

meant to avoid constant 2-squares whenever possible. It is of course analogous to the process of "completing constant elbows" discussed in Section 2. Let $A \in s(X)$. If some unassigned variable $x_{t}$ in $A$ lies in a 2-square $S$ whose three other entries are all equal in $A$ to some $\epsilon \in \mathbb{F}_{2}$, then set $x_{t}=\bar{\epsilon}$ in $A$ and define $\varphi(A) \in s(X)$ to be the resulting partially specialized array. In other words, we set

$$
\varphi(A)=\left.A\right|_{x_{t}=\bar{\epsilon}} .
$$

In order for this to be well-defined, we may choose $t$ to be the least index, if any, with the required property, and at the same time choose $S$ to be the lexicographically smallest corresponding 2-square. If there is no such index $t$, then we set

$$
\varphi(A)=A
$$

Note that, even if $A$ does not contain constant 2-squares, it may well happen that $\varphi(A)$ does. This will happen if $x_{t}$ lies in two 2-squares $S, S^{\prime}$, one having three 0 's in $A$, and the other having three 1 's in $A$.

Of course, the simple forcing procedure $\varphi$ may be iterated. Of special interest to us are the fixed points of $\varphi$. They are reached in a finite number of steps, depending of course on the initial argument $A$.

Now let $m \geq 0$, and denote by $E_{m}$ the set of all finite specializations $A \in s(X)$ with the following properties:

(1) The first $m$ variables $x_{1}, \ldots, x_{m}$ have been assigned 0 or 1 in $A$.

(2) $A$ is the final fixed point of the forcing procedure $\varphi$ applied iteratively to the element $A^{\prime}$ with all variables $x_{k}$ unassigned for $k \geq m+1$ and coinciding with $A$ for its first $m$ coefficients $x_{1}, \ldots, x_{m}$.

(3) $A$ contains no constant 2 -squares on its assigned entries.

Note that, for each $A \in E_{m}$, most variables $x_{i}$ with $i \geq m+1$ remain unassigned. But some of those variables may have been forced to a value 0 or 1 in $A$ in order to satisfy the required properties. Indeed, even if only $x_{1}, \ldots, x_{m}$ 
have been specialized at first, further variables might get specialized by successive applications of $\varphi$.

Observe that the set $E_{n^{2}}$ is in one-to-one correspondence with the set of all Erickson matrices of size $n \times n$.

The union $\bigcup_{m>0} E_{m}$ has the structure of a rooted plane tree, where the root is the unique element of $E_{0}$ having no assigned variables. Given an element $A \in E_{m}$, its immediate left (respectively right) successor, if it exists, is the unique element $\tilde{A}$ of $E_{m+1}$ which coincides with $A$ for $x_{1}, \ldots, x_{m}$ and satisfies $x_{m+1}=0$ (respectively $x_{m+1}=1$ ).

Note that the tree $\bigcup_{m \geq 0} E_{m}$ is finite. This follows from the fact that square Erickson matrices are uniformly bounded in size.

Our algorithm is now the classical depth-first algorithm for visiting all vertices of a finite rooted plane tree: start at the root in the direction of its leftmost child and continue walking turning always to the left at each bifurcation, respectively turning around and backtracking when hitting a leaf.

An important point is that this algorithm has only very small memory requirements: we do not need to store the tree $\bigcup_{m \geq 0} E_{m}$, we only store the currently used element in $E_{m}$ and use it to compute immediate successors or the unique immediate predecessor. This is crucial, since there happen to be more than $10^{11}$ Erickson matrices of size $9 \times 9$. (See below.)

Using the fact that the two subtrees issued from the two elements of $E_{1}$ (corresponding to $x_{1}=0$ and $x_{1}=1$ with all other entries unassigned) are mirrors of each other, we can halve the total amount of work by visiting only the subtree issued from the leftmost vertex (corresponding to $x_{1}=0$ ) of $E_{1}$. A further improvement is obtained by removing subtrees issued from solutions $A \in E_{n^{2}}$ such that $A$ is lexicographically after the transposed solution $A^{t}$. The resulting subset of leaves in $E_{n^{2}}$ then contains all Erickson matrices of size $n \times n$, up to transposition and up to flipping all coefficients.

\subsection{Outcome}

Denote by $\operatorname{Er}(n)$ the number of square Erickson matrices of size $n$ and with NW corner equal to 0 . Thus, the total number of square Erickson matrices of size $n$ is equal to $2 \operatorname{Er}(n)$. Running the above algorithm yields the following values of $\operatorname{Er}(n)$, for $n=2,3, \ldots, 15$. The square Erickson matrices of maximum size 14 will be described in the next section. 


\begin{tabular}{|r|r|}
\hline$n$ & \multicolumn{1}{|r|}{$\operatorname{Er}(n)$} \\
\hline 2 & 7 \\
3 & 138 \\
4 & 5490 \\
5 & 390856 \\
6 & 29169574 \\
7 & 1533415720 \\
8 & 29085496072 \\
9 & 156515895928 \\
10 & 54978562276 \\
11 & 2510360996 \\
12 & 1990028 \\
13 & 570132 \\
14 & 116114 \\
15 & 0 \\
\hline
\end{tabular}

\section{Extremal Erickson matrices}

The main computational result of this paper is Theorem 1.2, obtained with the backtracking algorithm of Section 3.2, and recalled here in a slightly different way.

Theorem 4.1. There exist no Erickson matrices of size $m \times n$ with $m \geq 14$ and $n \geq 15$. These bounds are sharp.

The next subsections exhibit extremal Erickson matrices, i.e. of size $13 \times$ $\infty$ and $14 \times 14$. In the latter case, we give a complete parametric description of all possible matrices up to automorphisms. This parametrization is then used in Section 4.3 to show that there are no Erickson matrices of size $14 \times 15$.

\subsection{An Erickson matrix of size $13 \times \infty$}

We now exhibit an Erickson matrix of size $13 \times \infty$. The first row has period 26 , repeating the sequence $s$ :

$$
s=(1,1,1,1,0,0,1,0,1,0,1,0,0,1,1,1,1,0,0,1,0,0,0,1,0,0) .
$$

Theorem 4.2. Let $A$ be the binary matrix indexed over $[13] \times \mathbb{Z}$ defined as follows: the first row of $A$ is 26-periodic repeating s, and each subsequent 
row is obtained by shifting the preceding one 5 units to the left. Then $A$ is a $13 \times \infty$ Erickson matrix.

This construction can be enlarged to get a doubly-infinite binary matrix containing no constant 2 -squares of span up to 13 . The reader might appreciate the Escher-like structure of the corresponding grid coloring. (See Figure 3.)

Theorem 4.3. Let $B$ be the doubly-infinite, doubly-periodic binary matrix of biperiod $26 \times 26$, indexed over $\mathbb{Z} \times \mathbb{Z}$, whose first row is 26-periodic repeating $s$, and where each row is obtained by shifting the preceding one 5 units to the left. Then B contains no constant 2-squares of span less than or equal to 13.

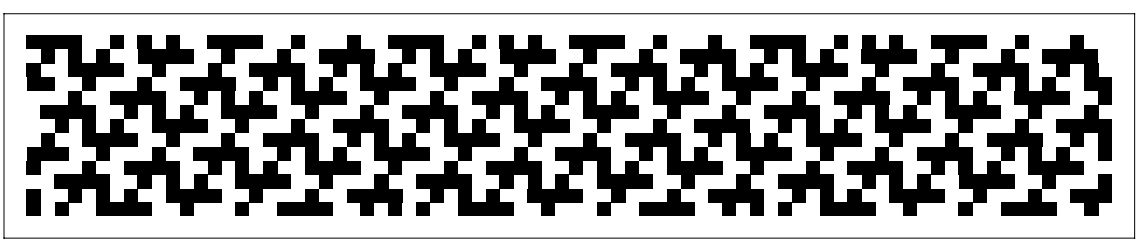

Figure 2: A $13 \times \infty$ Erickson matrix.

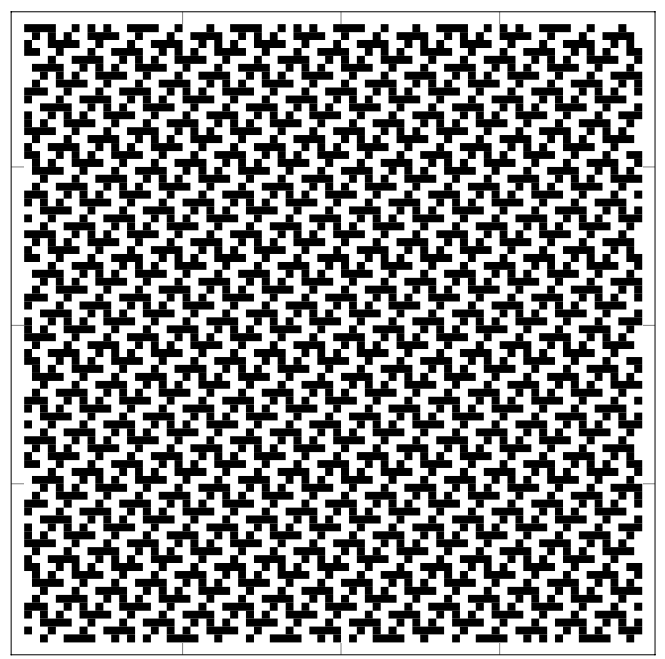

Figure 3: A binary coloring of $\mathbb{Z} \times \mathbb{Z}$ without constant 2-squares of span up to 13 . 
More Erickson matrices of size $13 \times \infty$ can be obtained from square Erickson tori of size 13. See Section 5.1.

\subsection{Erickson matrices of size $14 \times 14$}

We describe here the set of all extremal square Erickson matrices of size $14 \times 14$, harvested with the backtracking algorithm of Section 3.2.

For $n=14$, the total number of binary Erickson matrices of size $14 \times 14$ is equal to 232228 . These matrices are partitioned into exactly 14557 pairwise disjoint orbits under the automorphism group $G$. More precisely, these 14557 orbits divide up into exactly

- 14481 orbits of maximum size 16 ,

- 57 orbits of size 8 , and

- 19 orbits of size 4 .

The $8 \cdot 57$ Erickson matrices with a $G$-orbit of size 8 are all invariant under a half turn. The same is true for the $4 \cdot 19$ Erickson matrices with a $G$-orbit of size 4 , which are further invariant under flipping all entries followed by a $1 / 4$ turn or a $3 / 4$ turn.

For the record, Figure 4 displays the lexicographically smallest Erickson matrix of size $14 \times 14$.

We now list the complete set of 232228 square Erickson matrices of size $n=14$ up to automorphisms. They are partitioned into four families $A_{1}$, $A_{2}, A_{3}, A_{4}$.

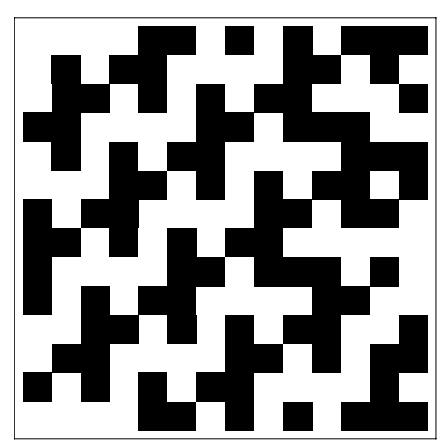

Figure 4: The lexicographically first square Erickson matrix of size 14. 


\subsubsection{The family $A_{1}$}

$$
A_{1}=\left(\begin{array}{cccccccccccccc}
x_{1} & 0 & 1 & 0 & 0 & 1 & 1 & 1 & 1 & 0 & 0 & 1 & 0 & x_{2} \\
1 & 1 & 1 & 1 & 0 & 0 & 1 & 0 & x_{3} & 0 & 1 & 0 & 0 & 1 \\
0 & 1 & 0 & x_{4} & 0 & 1 & 0 & 0 & 1 & 1 & 1 & 1 & 0 & 0 \\
1 & 0 & 0 & 1 & 1 & 1 & 1 & 0 & 0 & 1 & 0 & x_{5} & 0 & 1 \\
1 & 1 & 0 & 0 & 1 & 0 & x_{6} & 0 & 1 & 0 & 0 & 1 & 1 & 1 \\
0 & x_{7} & 0 & 1 & 0 & 0 & 1 & 1 & 1 & 1 & 0 & 0 & 1 & 0 \\
0 & 1 & 1 & 1 & 1 & 0 & 0 & 1 & 0 & x_{8} & 0 & 1 & 0 & 0 \\
0 & 0 & 1 & 0 & x_{9} & 0 & 1 & 0 & 0 & 1 & 1 & 1 & 1 & 0 \\
0 & 1 & 0 & 0 & 1 & 1 & 1 & 1 & 0 & 0 & 1 & 0 & x_{10} & 0 \\
1 & 1 & 1 & 0 & 0 & 1 & 0 & x_{11} & 0 & 1 & 0 & 0 & 1 & 1 \\
1 & 0 & x_{12} & 0 & 1 & 0 & 0 & 1 & 1 & 1 & 1 & 0 & 0 & 1 \\
0 & 0 & 1 & 1 & 1 & 1 & 0 & 0 & 1 & 0 & x_{13} & 0 & 1 & 0 \\
1 & 0 & 0 & 1 & 0 & x_{14} & 0 & 1 & 0 & 0 & 1 & 1 & 1 & 1 \\
x_{15} & 0 & 1 & 0 & 0 & 1 & 1 & 1 & 1 & 0 & 0 & 1 & 0 & x_{16}
\end{array}\right)
$$

Any binary assignment of the 16 variables in $A_{1}$ yields an Erickson matrix, provided

$$
\left(x_{1}, x_{2}, x_{15}, x_{16}\right) \neq(0,0,0,0), \quad\left(x_{1}, x_{2}, x_{15}, x_{16}\right) \neq(1,1,1,1) .
$$

Thus, the binary specializations of $A_{1}$ yield $14 \cdot 2^{12}=57344$ Erickson matrices. Under the action of the automorphism group $G$, they yield a total of 229376 Erickson matrices.

\subsubsection{The family $A_{2}$}

$$
A_{2}=\left(\begin{array}{cccccccccccccc}
1 & 1 & 1 & 1 & 0 & 0 & 1 & 0 & x_{1} & 0 & 1 & 0 & 0 & 0 \\
0 & 1 & 0 & x_{2} & 0 & 1 & 0 & 0 & 1 & 1 & 1 & 1 & 0 & 1 \\
1 & 0 & 0 & 1 & 1 & 1 & 1 & 0 & 0 & 1 & 0 & 1 & 0 & 0 \\
1 & 1 & 0 & 0 & 1 & 0 & x_{3} & 0 & 1 & 0 & 0 & 1 & 1 & 0 \\
0 & x_{4} & 0 & 1 & 0 & 0 & 1 & 1 & 1 & 1 & 0 & 0 & 1 & 1 \\
0 & 1 & 1 & 1 & 1 & 0 & 0 & 1 & 0 & 0 & 0 & 1 & 0 & 1 \\
0 & 0 & 1 & 0 & x_{5} & 0 & 1 & 0 & 0 & 1 & 1 & 1 & 1 & 1 \\
0 & 1 & 0 & 0 & 1 & 1 & 1 & 1 & 0 & 0 & 1 & 0 & 0 & 1 \\
1 & 1 & 1 & 0 & 0 & 1 & 0 & 1 & 0 & 1 & 0 & 0 & 1 & 0 \\
1 & 0 & x_{6} & 0 & 1 & 0 & 0 & 1 & 1 & 1 & 1 & 0 & 0 & 0 \\
0 & 0 & 1 & 1 & 1 & 1 & 0 & 0 & 1 & 0 & 0 & 0 & 1 & 1 \\
1 & 0 & 0 & 1 & 0 & 1 & 0 & 1 & 0 & 0 & 1 & 1 & 1 & 0 \\
x_{7} & 0 & 1 & 0 & 0 & 1 & 1 & 1 & 1 & 0 & 0 & 1 & 0 & 1 \\
1 & 1 & 1 & 1 & 0 & 0 & 1 & 0 & 1 & 0 & 1 & 0 & 0 & 0
\end{array}\right)
$$


Here the situation is simpler. Any binary assignment of the 7 variables in $A_{2}$ yields an Erickson matrix. This amounts to 128 Erickson matrices. As these matrices all have a $G$-orbit of size 16 , they yield a total of $2^{11}=2048$ Erickson matrices under the action of $G$.

\subsubsection{The family $A_{3}$}

$$
A_{3}=\left(\begin{array}{cccccccccccccc}
1 & 1 & 1 & 1 & 0 & 0 & 1 & 0 & x_{1} & 0 & 1 & 0 & 0 & 0 \\
0 & 1 & 0 & x_{2} & 0 & 1 & 0 & 0 & 1 & 1 & 1 & 1 & 0 & 1 \\
1 & 0 & 0 & 1 & 1 & 1 & 1 & 0 & 0 & 1 & 0 & 1 & 0 & 0 \\
1 & 1 & 0 & 0 & 1 & 0 & x_{3} & 0 & 1 & 0 & 0 & 1 & 1 & 0 \\
0 & 0 & 0 & 1 & 0 & 0 & 1 & 1 & 1 & 1 & 0 & 0 & 1 & 1 \\
0 & 1 & 1 & 1 & 1 & 0 & 0 & 1 & 0 & 0 & 0 & 1 & 0 & 1 \\
0 & 0 & 1 & 0 & 0 & 0 & 1 & 0 & 0 & 1 & 1 & 1 & 1 & 1 \\
0 & 1 & 0 & 0 & 1 & 1 & 1 & 1 & 0 & 0 & 1 & 0 & 0 & 1 \\
1 & 1 & 1 & 0 & 0 & 1 & 0 & 1 & 0 & 1 & 0 & 0 & 1 & 0 \\
1 & 0 & 1 & 0 & 1 & 0 & 0 & 1 & 1 & 1 & 1 & 0 & 0 & 0 \\
0 & 0 & 1 & 1 & 1 & 1 & 0 & 0 & 1 & 0 & 0 & 0 & 1 & 1 \\
1 & 0 & 0 & 1 & 0 & 1 & 0 & 1 & 0 & 0 & 1 & 1 & 1 & 0 \\
0 & 1 & 0 & 1 & 1 & 0 & 0 & 0 & x_{4} & 1 & 1 & 0 & 1 & x_{5} \\
1 & 1 & 1 & 1 & 0 & 0 & 1 & 0 & 1 & 0 & 1 & 0 & 0 & 0
\end{array}\right)
$$

Here again the situation is simple. Any binary assignment of the 5 variables in $A_{3}$ yields an Erickson matrix, with a $G$-orbit of size 16 . Thus, this family yields a total of $2^{9}=512$ Erickson matrices under automorphisms.

\subsubsection{The family $A_{4}$}

$$
A_{4}=\left(\begin{array}{cccccccccccccc}
x_{1} & 1 & 0 & 1 & 1 & 0 & 0 & 0 & x_{2} & 1 & 1 & 0 & 1 & x_{3} \\
0 & 0 & 0 & 1 & 0 & 1 & 0 & 1 & 0 & 0 & 1 & 1 & 1 & 0 \\
1 & 0 & 1 & 1 & 1 & 1 & 0 & 0 & 1 & 0 & 0 & 0 & 1 & 1 \\
0 & 0 & 1 & 0 & 1 & 0 & 0 & 1 & 1 & 1 & 1 & 0 & 0 & 0 \\
0 & 1 & 1 & 0 & 0 & 1 & 0 & 1 & 0 & 1 & 0 & 0 & 1 & 0 \\
x_{4} & 1 & 0 & 0 & 1 & 1 & 1 & 1 & 0 & 0 & 1 & 0 & 0 & 1 \\
1 & 0 & 1 & 0 & 0 & 0 & 1 & 0 & 0 & 1 & 1 & 1 & 1 & 1 \\
1 & 1 & 1 & 1 & 1 & 0 & 0 & 1 & 0 & 0 & 0 & 1 & 0 & 1 \\
1 & 0 & 0 & 1 & 0 & 0 & 1 & 1 & 1 & 1 & 0 & 0 & 1 & x_{5} \\
0 & 1 & 0 & 0 & 1 & 0 & 1 & 0 & 1 & 0 & 0 & 1 & 1 & 0 \\
0 & 0 & 0 & 1 & 1 & 1 & 1 & 0 & 0 & 1 & 0 & 1 & 0 & 0 \\
1 & 1 & 0 & 0 & 0 & 1 & 0 & 0 & 1 & 1 & 1 & 1 & 0 & 1 \\
0 & 1 & 1 & 1 & 0 & 0 & 1 & 0 & 1 & 0 & 1 & 0 & 0 & 0 \\
x_{6} & 1 & 0 & 1 & 1 & x_{7} & 0 & 0 & 0 & 1 & 1 & 0 & 1 & x_{8}
\end{array}\right)
$$


In the present case, a binary assignment of the 8 variables in $A_{4}$ yields an Erickson matrix if and only if

$$
\begin{array}{r}
\left(x_{1}, x_{3}, x_{6}, x_{8}\right) \neq(0,0,0,0),\left(x_{1}, x_{3}, x_{6}, x_{8}\right) \neq(1,1,1,1), \\
(3) \quad\left(x_{1}, x_{2}\right) \neq(1,1),\left(x_{7}, x_{8}\right) \neq(1,1),\left(x_{3}, x_{5}\right) \neq(0,0),\left(x_{4}, x_{6}\right) \neq(0,0) .
\end{array}
$$

Because of symmetries in $A_{4}$, the orbits of its various specializations attain sizes 4, 8 and 16. This family, under the above affine constraints and the action of $G$, yields a total of 292 Erickson matrices.

Note that the matrices $A_{1}$ and $A_{4}$ are invariant under a $1 / 4$ turn followed by a suitable permutation of indices and the flipping of all entries.

4.2.5. Summary Summarizing the above, we obtain the following complete description of square Erickson matrices of size $n=14$.

Theorem 4.4. A binary square matrix of size 14 is an Erickson matrix if and only if it is equivalent, up to automorphisms, to a binary specialization of either $A_{1}$ under constraints (1), or $A_{2}$, or $A_{3}$, or $A_{4}$ under constraints (2,3). There are exactly 232228 square Erickson matrices of size 14, where

$$
232228=229376+2048+512+292
$$

according to the partition into types $A_{1}, A_{2}, A_{3}$ and $A_{4}$.

4.2.6. What about linear algebra? It is natural to wonder whether Erickson matrices have any special linear algebraic properties. A first exploration of this question did not reveal much so far. Let us only mention a few observations:

- All $14 \times 14$ Erickson matrices have rank equal to 13 or 14 .

- Among all determinants of $14 \times 14$ Erickson matrices, one finds $3^{8}$ and $5^{5}$.

- The determinant of $A_{3}$ is much simpler than for $A_{1}, A_{2}, A_{4}$. It is equal to

$$
\begin{aligned}
3\left(x_{1}-1\right)( & 990+35 x_{2}-90 x_{3}-495 x_{5}-80 x_{2} x_{3}-123 x_{2} x_{5}+45 x_{3} x_{5} \\
+ & \left.23 x_{2} x_{3} x_{5}\right) .
\end{aligned}
$$

- The characteristic polynomial of $A_{4}$ is divisible by $\left(X^{2}-5\right)\left(X^{2}+3\right)$. 


\subsection{The case of size $14 \times 15$}

For the proof of Theorem 4.1 to be complete, it remains to show that there are no Erickson matrices of size $14 \times 15$. This can easily be checked as follows, by using our parametric description of Erickson matrices of size $14 \times 14$. Up to reversion and up to flipping all entries, all four vectors corresponding to the first and last rows and the first and last columns of a $14 \times 14$ Erickson matrix are of the following type:

$$
\begin{aligned}
& (0,0,0,0,1,1,0,1, *, 1,0,1,1,1), \\
& (0,1,0,0,1,1,1,1,0,0,1,0, *, 0), \\
& \left(x_{1}, 0,1,0,0,1,1,1, x_{2}, 0,0,1,0, *\right),
\end{aligned}
$$

with $\left(x_{1}, x_{2}\right) \neq(0,0)$ and with $*$ an arbitrary element of $\{0,1\}$. The first two rows arise in the families $A_{2}$ and $A_{3}$, and the last row occurs in $A_{4}$ and with $x_{2}$ assigned to 1 in $A_{1}$.

The presumed existence of a $14 \times 15$ Erickson matrix would imply that one of these rows arises as the second or second-to-last row or the second or second-to-last column of a $14 \times 14$ Erickson matrix. However, a direct inspection on $A_{1}, A_{2}, A_{3}, A_{4}$ shows that this is not the case, thereby settling our claim.

\section{Related structures}

Here we discuss some variants of the notion of Erickson matrices.

\subsection{Erickson tori of square size}

Besides 2-colorings of the grids $[m] \times[n]$, it is also interesting to consider 2-colorings of toroidal grids. This amounts to binary matrices with indices in $\mathbb{Z} / m \mathbb{Z} \times \mathbb{Z} / n \mathbb{Z}$. We define an Erickson torus of size $m \times n$ to be an array indexed by $\mathbb{Z} / m \mathbb{Z} \times \mathbb{Z} / n \mathbb{Z}$, containing no constant 2 -squares with indices of the form $\{(i, j),(i+t, j),(i, j+t),(i+t, j+t)\} \subset \mathbb{Z} / m \mathbb{Z} \times \mathbb{Z} / n \mathbb{Z}$ for $t=1, \ldots, \min (n, m)-1$. 
For each $n \geq 2$, the number $\tau(n)$ of Erickson tori of square size $n \times n$ is given by the following computer-constructed table.

\begin{tabular}{|c|r|}
\hline$n$ & $\tau(n)$ \\
\hline 2 & 14 \\
3 & 156 \\
4 & 6112 \\
5 & 114040 \\
6 & 878448 \\
7 & 360360 \\
8 & 160416 \\
9,10 & 0 \\
11 & 180224 \\
12 & 0 \\
13 & 427336 \\
$\geq 14$ & 0 \\
\hline
\end{tabular}

Of course, Erickson tori give rise to Erickson matrices. However, they behave somewhat differently. In particular, subarrays of Erickson tori are not generally Erickson tori of smaller size. This is a partial explanation of the gaps for $n=9,10,12$ in the table above. Moreover, the group of automorphisms acting on Erickson tori of square size is larger than the group of automorphisms acting on Erickson matrices. It contains of course all 16 elements preserving Erickson matrices, but it also contains all shifts of indices corresponding to cyclic permutations of rows and columns. Moreover, in the case of Erickson tori of square size $n \times n$, we get all dilatations

$$
a_{i, j} \longmapsto a_{\lambda i, \lambda j}
$$

with $\lambda \in(\mathbb{Z} / n \mathbb{Z})^{*}$.

Erickson tori of square size $n \times n$ give rise to doubly periodic infinite binary arrays containing no constant 2 -squares of span at most $n$. Every row and column of such an array defines an $n$-periodic binary sequence.

A particularly nice class of examples is given by Erickson tori associated to $n$-periodic row-sequences differing only by shifts of their indices. Such an Erickson torus of size $n \times n$ is thus completely described (up to a translation of its indices) by a row-period $\left(\alpha_{0}, \alpha_{1}, \ldots, \alpha_{n-1}\right) \in\{0,1\}^{n}$ and by the shift $\kappa$ relating two adjacent rows. Reading indices modulo $n$, the associated infinite 
array is thus

$$
\begin{array}{cccccccc} 
& \vdots & \vdots & \vdots & & & & \\
\ldots & \alpha_{0} & \alpha_{1} & \alpha_{2} & \ldots & \alpha_{n-1} & \alpha_{0} & \ldots \\
\ldots & \alpha_{\kappa} & \alpha_{1+\kappa} & \alpha_{2+\kappa} & \ldots & \alpha_{n-1+\kappa} & \alpha_{\kappa} & \ldots \\
\ldots & \alpha_{2 \kappa} & \alpha_{1+2 \kappa} & \ldots & & & & \\
& \vdots & \vdots & & & & &
\end{array}
$$

Note that replacing the shift value $\kappa$ by $n-\kappa$ gives rise to an isomorphic solution.

Examples of such "sequential" Erickson tori of square size exist for all $n$ for which $\tau(n) \neq 0$. The following table gives for each such $n$ a full period $\left(\alpha_{0}, \ldots, \alpha_{n-1}\right) \in\{0,1\}^{n}$ together with the value $\kappa$ indicating the shift of indices between adjacent rows.

\begin{tabular}{|r|l|l|}
\hline \multicolumn{1}{|c|}{$\left(\alpha_{0}, \alpha_{1}, \ldots, \alpha_{n-1}\right)$} & $\kappa$ \\
\hline 2 & $(0,1)$ & 1 \\
3 & $(0,1,1)$ & 1 \\
4 & $(0,0,1,1)$ & 1,2 \\
5 & $(0,0,1,0,1)$ & 2 \\
6 & $(0,0,0,1,1,1)$ & 2 \\
7 & $(0,0,0,1,0,1,1)$ & 2,3 \\
8 & $(0,0,1,0,1,1,0,1)$ & 3 \\
11 & $(0,0,0,1,0,0,1,0,1,1,1)$ & 2,5 \\
13 & $(0,0,0,1,0,0,1,1,1,1,0,0,1)$ & 5 \\
\hline
\end{tabular}

\subsection{Gallai's theorem}

The presence of a constant 2-square in any binary square matrix of sufficiently large size follows from a $d$-dimensional generalization of van der Waerden's theorem due to Tibor Gallai in the 1930's. See e.g. [1] or [12, page 40]. Our attempt to locate the first written mention of this theorem revealed a somewhat confused situation, which we have tried to clarify and put in historical context in the last section. See also [17, Chap. 42]. Here is Gallai's theorem as stated in [1], where a nice proof is included.

Theorem 5.1. Let $S$ be a finite subset of $\mathbb{N}^{d}$. For any $k$-coloring of $\mathbb{N}^{d}$, there is a positive integer $a \geq 1$ and a point $v \in \mathbb{N}^{d}$ such that the set $a S+v-i . e .$, some translation of some dilation of $S$ - is monochromatic. Furthermore, the dilation factor $a$ and the coordinates of the translation 
point $v$ are bounded by a function that depends only upon the set $S$ and the number $k$ (and not upon the particular coloring used).

Erickson matrices correspond to the case $d=k=2$ and

$$
S=\{(0,0),(0,1),(1,0),(1,1)\}
$$

A simpler case, still with $d=k=2$ but with $S=\{(0,0),(1,0),(0,1)\}$, corresponds to what might be called Erickson triangles. The maximum size of such a triangle without constant sub-triangle can be determined by hand, and is equal to 4 . Thus, every binary triangular array

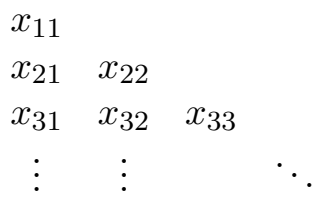

of size at least 5 contains a constant subtriangle of the form $x_{i j}=x_{(i+t) j}=$ $x_{(i+t)(j+t)}$. See e.g. [2, Lemma 1].

More generally, for $s \geq 2$, we define an $s$-square in $[n] \times[n]$ to be any $s \times s$ square subgrid of the form

$$
\{i, i+t, \ldots, i+(s-1) t\} \times\{j, j+t, \ldots, j+(s-1) t\}
$$

with $s^{2}$ points. For any positive integer $k \geq 2$, let us denote by $n(k, s)$ the smallest integer $n$ for which any $k$-coloring of the grid $[n]^{2}$ contains a monochromatic $s$-square. Gallai's theorem ensures that $n(k, s)$ is finite. It would be interesting to understand the behavior of $n(k, s)$ beyond the case $k=s=2$, for which $n(2,2)=15$ by Theorem 1.2. The next interesting numbers to determine are

- $n(3,2)$, forcing constant 2-squares for any 3-coloring of $[n(3,2)]^{2}$,

- $n(2,3)$, forcing constant 3 -squares for any 2-coloring of $[n(2,3)]^{2}$.

Note. While this paper was being refereed, colleagues in computer science succeeded in establishing the lower bound

$$
n(2,3) \geq 663
$$

They did so by constructing, with the technique of simulated annealing, a square binary matrix of size 662 without constant 3-squares [4]. 
These questions may of course be generalized to any dimension $d \geq 3$. Besides constant "hypercubes", the simplest non-trivial case for $d=3$ is given by

$$
S=\{(0,0,0),(1,0,0),(0,1,0),(0,0,1)\}
$$

and gives rise to what might be called "3-dimensional Erickson simplices." Here again, it would be most interesting to determine the smallest $n$ forcing the presence of a monochromatic Erickson simplex in a binary cube of size $n \times n \times n$.

\subsection{Historical remarks}

We take this opportunity to try to clarify some bibliographical and historical details, resulting from our attempt to locate the first written source of Gallai's theorem. Tibor Gallai himself apparently never published it. Rather, he told it to Richard Rado, who seemingly first stated it in [16]. (But not in his earlier paper [15], as mistakenly stated ${ }^{2}$ in [8].) A prior and weaker version of Gallai's theorem, yet still generalizing van der Waerden's theorem to $d$ dimensions, was obtained by Rado in [14]. This weaker version states the following: Given $k, l, d \geq 1$, for any $k$-coloring of the grid $[n]^{d}$ with $n$ sufficiently large, there is a monochromatic subset of the form $A_{1} \times \cdots \times A_{d}$, where each $A_{i}$ is an arithmetic progression of length $l$. Coming back to Gallai's theorem proper, Rado writes in [16, p. 123]:

"This extension [of van der Waerden's theorem] was first proved by Dr. G.

Grünwald, who kindly communicated it to me."

Yes, Grünwald is the original name of Gallai. The change from Germansounding to more Hungarian-sounding names was frequent for Hungarian Jews up to the 1930's, in a context of social pressure and harsh antisemitism $[3,18]$. Yet the initial " $G$ " in the above quotation is quite mysterious and confusing, as it should have been "T" for Tibor. Indeed, there is another Hungarian-Jewish mathematician called Géza Grünwald, not a relative but a close friend of Tibor Grünwald, and who died at age 31 in tragic circumstances during WWII [3, 18]. Another source of confusion comes from the fact that both Tibor and Géza Grünwald wrote joint papers with their common friend Paul Erdös. However, there is no controversy at all, and Tibor Grünwald is the author of Gallai's theorem. We will probably never know why Rado wrote "G. Grünwald" rather than "T. Grünwald" in [16]. It

\footnotetext{
${ }^{2}$ Ron Graham kindly confirmed this to us.
} 
might simply be a typo, as suggested in Soifer's book [17], whose chapter 42 is dedicated to Gallai's theorem. See $[5,7]$ for moving obituaries of Gallai. See also [6].

\section{Acknowledgements}

We are most grateful to M. Erickson for his comments on earlier versions of this paper, and to B. Landmann, L. Lovász, R. Graham and V.T. Sós for their help with bibliographical and historical details. Both referees are thanked for their extremely careful reading of the manuscript.

\section{References}

[1] P. G. Anderson, A Generalization of Baudet's Conjecture (Van Der Waerden's Theorem), Amer. Math. Monthly 83 (1976) 359-361. MR0396292

[2] M. Axenovich, J. Manske, On monochromatic subsets of a rectangular grid, Integers: Electronic Journal of Combinatorial Number Theory 8 (2008), \#A21. MR2425619

[3] L. Babai, In and out of Hungary: Paul Erdös, his friends, and times, in Combinatorics, Paul Erdős is eighty, Vol. 2 (Keszthely, 1993), 7-95, Bolyai Soc. Math. Stud., 2, János Bolyai Math. Soc., Budapest, 1996. Also available online at http://www.cs.uchicago.edu/research/ publications/techreports/TR-2001-03. MR1395855

[4] D. Robilliard, A. Boumaza, V. Marion-Poty, Meta-Heuristic Search and Square Erickson Matrices, to appear in the Proceedings of the 2010 IEEE Congress on Evolutionary Computation (IEEE World Congress on Computational Intelligence). Barcelona, Spain. IEEE 2010. Available online at http://hal .archives-ouvertes.fr/ hal-00448392/en/.

[5] L. Babai, V. T. Sós, Tibor Gallai, 1912-1992, Combinatorica 12 (1992) 371-372. MR1194726

[6] P. Erdős, Personal reminiscences and remarks on the mathematical work of Tibor Gallai, Combinatorica 2 (1982) 207-212. MR0698647

[7] P. Erdős, In memory of Tibor Gallai, Combinatorica 12 (1992) 373-374. MR1194727 
[8] P. Erdős, R. L. Graham, Old and new problems and results in combinatorial number theory: van der Waerden's theorem and related topics, Enseign. Math. 25 (1979) 325-344. MR0570317

[9] M. J. Erickson, Introduction to Combinatorics, Wiley-Interscience Series in Discrete Mathematics and Optimization, 1996. MR1409365

[10] W. T. Gowers, A new proof of Szemerédi's theorem, Geom. Funct. Anal. 11 (2001) 465-588. MR1844079

[11] R. Graham, http://www.math.ucsd.edu/ sbutler/ron/papers. html

[12] R. L. Graham, B. L. Rothschild, J. H. Spencer, Ramsey Theory. WileyInterscience Series in Discrete Mathematics and Optimization. John Wiley \& Sons Inc., New York, second edition, 1990. MR1044995

[13] M. Kouril, J. L. Paul, The van der Waerden number W(2,6) is 1132, Experiment. Math. 17 (2008) 53-61. MR2410115

[14] R. Rado, Verallgemeinerung eines Satzes von der Waerden mit Anwendungen auf ein Problem der Zahlentheorie, Sitzungsberichte Acad. Berlin (1933) 589-596.

[15] R. Rado, Studien zur Kombinatorik, Math. Zeit. 36 (1933) 424-480. MR1545354

[16] R. Rado, Note on combinatorial analysis, Proc. London Math. Soc (1943) 122-160. MR0009007

[17] A. Soifer, The mathematical coloring book. Mathematics of coloring and the colorful life of its creators. Springer, New York, 2009. ISBN: 978-0-387-74640-1. MR2458293

[18] V. T. Sós, personal communication, June 2009.

ROLAND BACHER

INSTITUT FOURIER

LABORATOIRE DE MATHÉMATIQUeS

UMR 5582 (UJF-CNRS)

BP 74, 38402 St Martin D'Hères Cedex

FRANCE

E-mail address: Roland.Bacher@ujf-grenoble.fr 
Shalom Eliahou

Univ Lille Nord de France

F-59000 LILLE

FRANCE

ULCO, LMPA J. LIOUVILLE

B.P. 699, F-62228 CALAIS

FRANCE

CNRS, FR 2956

FRANCE

E-mail address: eliahou@lmpa.univ-littoral.fr

Received September 3, 2009 